\title{
Synthesis of 2-Hydroxyethyl Methacrylate-Dimethylsiloxane Block Copolymers and Their Ability to Suppress Blood Platelet Aggregation
}

\author{
Masashi ShImAda, Masaru MiYaHARA, Hiroshi TAHARA, \\ Isao SHINOHARA, Teruo OKANO, ${ }^{*}$ Kazunori KATAOKA, ${ }^{*}$ \\ and Yasuhisa SAKURAI* \\ Department of Polymer Chemistry, Waseda University, \\ Okubo, Shinjuku-ku, Tokyo 160, Japan \\ *Institute of Medical Engineering, and Heart Institute of Japan, \\ Tokyo Women's Medical College, \\ Kawadacho, Shinjuku-ku, Tokyo 162, Japan
}

(Received March 17, 1983)

\begin{abstract}
In order to elucidate the interaction between blood platelets and polymer surfaces of microdomain structure, block copolymers consisting of hydrophilic chains of 2hydroxyethyl methacrylate (HEMA) and hydrophobic chains of dimethylsiloxane (DMS) were synthesized. Films of the HEMA-DMS block copolymers exhibiting hydrophilic-hydrophobic microphase-separated structures were found to enhance platelet adhesion more than homogeneous surfaces of poly(HEMA) or poly(DMS). Platelet adhesion to the block copolymer increased with morphological changes in microdomains so long as the HEMA composition of the block copolymer was 0.90 to 0.58 . Platelet adhesion also varied with morphological change in the microdomains caused by changing the casting solvents, though an identical copolymer was employed. These results indicated that platelet adhesion to the HEMA-DMS block copolymer was influenced by domain morphology rather than HEMA composition of the copolymers. In spite of the enhancement of platelet adhesion, the HEMA-DMS block copolymers effectively suppressed shape change and aggregation of adhered platelets. The HEMA-DMS block copolymers were considered to have good antithrombogenecity on the basis of inhibition of activation and aggregation of adhered platelets.

KEY WORDS 2-Hydroxyethyl Methacrylate-Dimethylsiloxane Block Copolymer / Microdomain Structure / Platelet Adhesion / Antithrombogenecity /
\end{abstract}

The antithrombogenic property of synthetic polymers is influenced remarkably by the balance between hydrophilicity and hydrophobicity of polymer surface. ${ }^{1}$ We propose that the microdomain structure of polymer surfaces constructed of hydrophilic and hydrophobic sites of orderly assembled marcomolecules is the most important factor for designing effective antithrombogenic polymers. ${ }^{2}$

We synthesized new amphiphilic block copolymers and comb type graft compolymers consisting of a hydrophilic monomer, 2-hydroxyethyl methacrylate (HEMA), and a hydrophobic monomer, sty- rene (St). ${ }^{3}$ Films of HEMA-St block copolymers formed hydrophilic-hydrophobic microdomain structures on their surfaces. Platelet adhesion, an important process in the initial stage of thrombus formation, was studied in relation to block copolymer surfaces. The amount and deformation of adhered platelets were effectively suppressed on the surface of HEMA-St block and graft copolymers. This suppressing effect was found to be influenced by the shape and size of the microdomain rather than by the composition of the copolymers. ${ }^{2,4}$ The copolymers having microdomain structures of alternate lemellae with about $0.05 \mu \mathrm{m}$ in width exhibited 
the least adhesion and aggregation. The balance between hydrophilicity and hydrophobicity of the surface of the hydrophilic-hydrophobic microdomain structure can be changed by varying the surface free energy of the microdomains. In this paper, we synthesized new block copolymers constructed of hydrophilic chains of HEMA and hydrophobic chains of dimethylsiloxane (DMS) having a lower surface free energy than polystyrene (PSt). The HEMA-DMS block copolymer films formed surfaces of hydrophilic and hydrophobic microdomain structure. The surface free energy gap between hydrophilic and hydrophobic microdomains of the HEMA-DMS block copolymer is larger than that of the HEMA-St block copolymer, since the DMS chain is more hydrophobic than the St chain. These HEMA-DMS block copolymers are suitable models for investigating the influence of surface free energy gap on the adhesion behaviour of platelets. We estimated the platelet adhesivity and shape change of adhered platelets on the HEMA-DMS block copolymers. From the results, the role of hydrophilic-hydrophobic microdomains in interaction of platelets with the copolymer surfaces is discussed.

\section{EXPERIMENTAL}

\section{Synthesis of HEMA-DMS Block Copolymers}

The HEMA-DMS block copolymers were synthesized by a coupling reaction of the oligomers of amino-telechelic oligo(HEMA) and isocyanatetelechelic oligo(DMS). Oligo(DMS) having two isocyanate groups at each end of the chain was prepared as follows. Hydroxyl-telechelic oligo-DMS (Petrarch Systems, Inc., U.S.A., functionality; 2.0) was allowed to react with toluene diisocyanate (TDI) in toluene at $0^{\circ} \mathrm{C}$ for $48 \mathrm{~h}$. The quantitative introduction of isocyanate groups to both ends of the oligo(DMS) under this condition was confirmed by IR and isocyanate group titration. Aminotelechelic oligo(HEMA), which has one amino group at the chain end, was synthesized by the polymerization of HEMA using 2-aminoethanethiol as a chain transfer agent, as described elsehwere. ${ }^{3,5}$

The HEMA-DMS block copolymers were synthesized by reacting the isocyanate-telechelic oligo(DMS) with amino-telechelic oligo(HEMA) under the condition of $[\mathrm{NCO}] /\left[\mathrm{NH}_{2}\right]=1.0$ in dimethylformamide (DMF) at $0^{\circ} \mathrm{C}$ for $96 \mathrm{~h}$. The reaction mixture was precipitated with diethyl ether to remove the unreacted oligo(DMS) at first, the precipitated polymer was then dissolved in DMF-toluene and reprecipitated with a methanol-water mixture to remove the unreacted oligo-HEMA.

\section{Analysis of the Microphase-Separated Structure of Block Copolymers}

The block copolymers were dissolved in pyridine or a DMF-toluene mixture. The concentration of the solution was adjusted to $0.5 \mathrm{wt} \%$. The solution was cast on a collodion membrane coated with a carbon layer and stored at $40^{\circ} \mathrm{C}$ for $24 \mathrm{~h}$ to evaporate the solvent. The prepared films of the block copolymers were then stained with osmium tetraoxide vapor at $40^{\circ} \mathrm{C}$ for $24 \mathrm{~h}$ in a desiccated glass container. The microphase-separated structures of the films of the block copolymers were observed by a transmission electron microscope (Hitachi, HU11).

\section{Estimation of the Interaction between Polymers and} Platelets

The block copolymers were coated on glass beads (40-60 mesh, Japan Chromato. Ind.) by a solventevaporation technique. ${ }^{6}$ That is, the block copolymer solution was cast on the surface of the glass beads which were then stored at $40^{\circ} \mathrm{C}$ for $24 \mathrm{~h}$ to evaporate the solvent and then dried under reduced pressure. The beads coated with the polymers were closely packed in a column (inner diameter; $3 \mathrm{~mm}$, length; $10 \mathrm{~cm}$ ) fitted with a three-way cock at the column outlet. Canine whole blood extructed from juglar veins without anticoagulants was injected into the column at a flow rate of $1.2 \mathrm{ml} / \mathrm{min}$ for 1 min using a perfusion pump (Precidol, Model 5003). The column was previously primed with a saline solution to avoid the direct contact of blood with air. Eluted blood from the column was collected in a bottle containing etylenediaminetetraacetic acid (EDTA) and the number of platelets was counted in order to estimate the retention of platelets in the column. ${ }^{7,8}$ After this, the column was rinsed with a saline solution at a flow rate of $1.9 \mathrm{ml} \mathrm{m^{-1 }}$ for $1 \mathrm{~min}$. The beads packed $2 \mathrm{~cm}$ downward from the inlet were taken out and fixed with a $1.25 \%$ of glutaraldehyde-saline solution. The beads specimens were freeze-dried, and then subjected to scanning electron microscopy observation (Hitachi-Akashi, MSM-1001). 
Adhesion Behaviour of Platelets on Block Copolymers

Table I. Synthesis of HEMA-DMS block copolymers

\begin{tabular}{|c|c|c|c|c|c|}
\hline \multirow{2}{*}{ No. } & \multirow{2}{*}{$\begin{array}{l}\text { Chain length of } \\
\text { HEMA }\left(M_{n}\right)^{\mathrm{a}}\end{array}$} & \multirow{2}{*}{$\begin{array}{l}\text { Chain length of } \\
\text { DMS }\left(M_{n}\right)^{\mathrm{b}}\end{array}$} & \multicolumn{2}{|c|}{$\begin{array}{l}\text { Mole fraction of HEMA } \\
\text { in the block copolymer }\end{array}$} & \multirow{2}{*}{$\frac{\text { Yield }^{\mathrm{d}}}{\%}$} \\
\hline & & & Calcd & Found $^{c}$ & \\
\hline$[1]$ & 2,700 & 2,400 & 0.56 & 0.58 & 30.3 \\
\hline [2] & 3,100 & 2,400 & 0.60 & 0.61 & 30.4 \\
\hline$[3]$ & 4,200 & 2,400 & 0.67 & 0.67 & 67.6 \\
\hline$[4]$ & 5,100 & 2,400 & 0.71 & 0.70 & 38.1 \\
\hline$[5]$ & 7,100 & 2,400 & 0.77 & 0.82 & 17.4 \\
\hline$[6]$ & 12,100 & 2,400 & 0.85 & 0.90 & 19.8 \\
\hline
\end{tabular}

a Determined by amino group titration.

b Determined from the concentration of hydroxyl groups.

c Calculated from $\mathrm{C} \%$ of elemental analysis.

d Yield of the coupling reaction of oligo(HEMA) and oligo(DMS.)

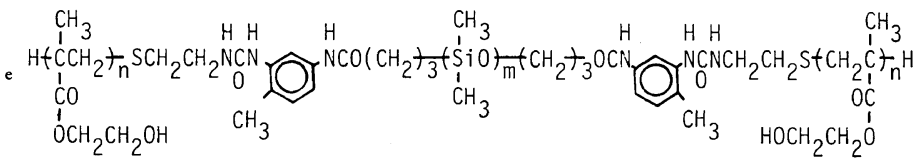

\section{RESULTS AND DISCUSSION}

\section{HEMA-DMS Block Copolymers}

The results of the preparation of HEMA-DMS block copolymers are shown in Table I. The calculated composition of HEMA in the block copolymer was approximately equal to that determined by elemental analysis. The presence of HEMA in the block copolymer was demonstrated by the carbonyl band at $1740 \mathrm{~cm}^{-1}$, the hydroxyl group band at $3450 \mathrm{~cm}^{-1}$, and many other absorptions in the IR spectra. DMS was also contained in the block copolymers as indicated by the absorption of Si-C stretching vibration at $800 \mathrm{~cm}^{-1}$. The increase in molecular weight in the block copolymers was confirmed by the rise in intrinsic viscosity $([\eta]=$ 0.37, blcok copolymers [3] of Table I) compared with prepolymers of oligo(HEMA) $([\eta]=0.29)$ and oligo(DMS) $([\eta]=0.052)$ in a DMF-toluene $(4: 6)$ mixed solvent at $25^{\circ} \mathrm{C}$. The gel-permeation chromatogram of the block copolymers was obtained as a single peak, indicating that the unreacted prepolymers of oligo(HEMA) and oligo(DMS) were eliminated in the precipitation process.

\section{Microphase-Separated Structure of HEMA-DMS Block Copolymers}

The HEMA-DMS block copolymer composed of hydrophilic chains of HEMA and hydrophobic chains of DMS, exhibited the microphase-separated structure shown in Figure 1. The films of the block copolymers were cast from a mixed solution of DMF-toluene (4:6). The HEMA domains in the block copolymers were stained with osmium tetroxide. The film of the HEMA-DMS block copolymer of the 0.61 HEMA mole fraction [2] showed a morphology of isolated HEMA domains in a continuous domain of DMS, as shown in Figure 1-A. The microphase-separated structure of the block copolymer containing the 0.67 mole fraction of HEMA [3] showed the continuous domains of HEMA and DMS in Figure 1-B. This is a transitional pattern from Figure 1-A to 1-C, whereas the morphology of dispersed DMS domains in the continuous domain of HEMA was observed in the block copolymer of the 0.82 mole fraction of HEMA [5].

In the case of the film of HEMA-DMS block copolymer [3] cast from a pyridine solution, dispersed domains of HEMA in the continuous domain of DMS were observed as shown in Figure 2. Figures 1-B and 2 show that the pattern of the microdomain structure of the block copolymer [3] was altered by changing the casting solvents. The morphology of the microdomain can be regulated by adopting appropriate casting solvents as well as 


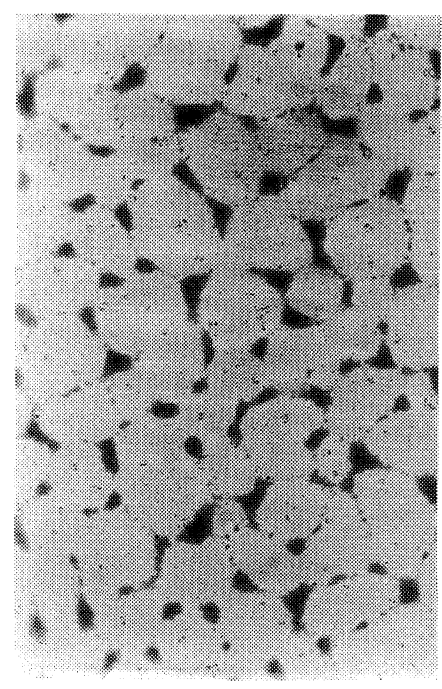

A

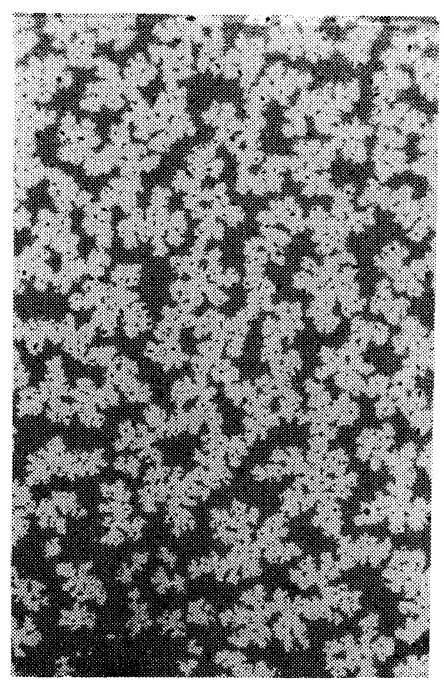

B

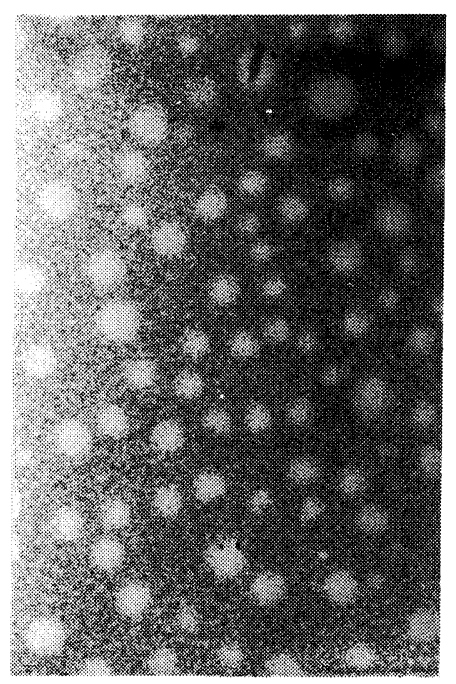

C

$2 \mu$

Figure 1. Electron micrographs of the microphase-separated structure of HEMA-DMS block copolymers cast from a DMF-toluene $(4: 6)$ solution and stained with osmium tetroxide. Mole fraction of HEMA: A, (0.61) [2]; B, (0.67) [3]; C, (0.82) [5].

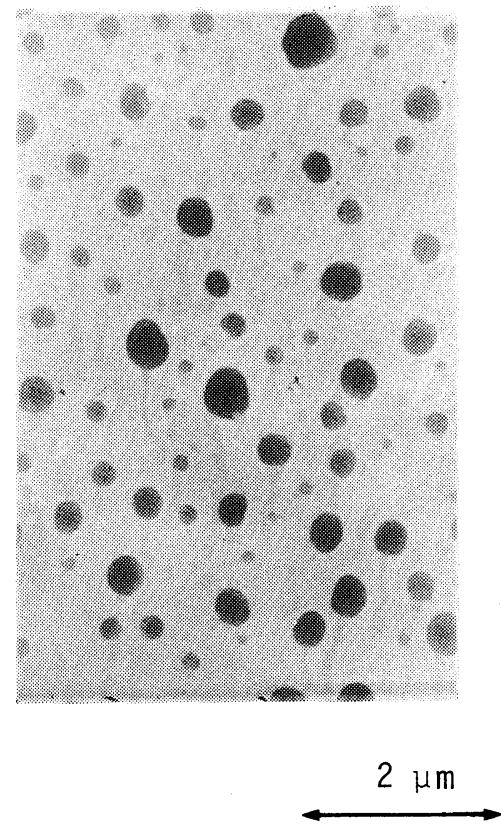

Figure 2. Electron micrograph of the microphase-separated structure of the HEMA-DMS block copolymer cast from a pyridine solution and stained with osmium tetroxide. Mole fraction of HEMA: 0.67 [3]. changing the composition of the block copolymers

Adhesion Behaviour of Platelets on the Surface of HEMA-DMS Block Copolymer

Platelet adhesion to the surface of HEMA-DMS block copolymer is shown in Figure 3 compared with that of the homopolymer surfaces. Fractions of about 30 to $40 \%$ of the platelets from whole blood adhered to both the hydrophilic surface of poly(HEMA) (PHEMA) and hydrophobic surface of poly(DMS) (PDMS) whose surface free energy was $18 \mathrm{erg} \mathrm{cm}^{-1}$. We also comfirmed that PSt, whose surface free energy was $32 \mathrm{erg} \mathrm{cm}^{-1}$, showed the same amount of platelet adhesion as PDMS or PHEMA. ${ }^{9}$ On the other hand, the HEMA-DMS block copolymer was found to enhance platelet adhesion compared with PHEMA and PDMS. That is, platelet adhesion increased with an decrease in the HEMA mole fraction in the range of 1.0 to 0.58 . These results suggest that platelet adhesion is influenced by the morphology of the microdomain of the block copolymer rather than the surface free energy of the polymers.

As for the shape of the adhered platelets, significant differences were found on block copolymer and homopolymer surfaces. On PHEMA surface, 
platelets extruded many pseudopods and aggregated with each other, whereas the platelets were largely deformed and spread on the PDMS surface

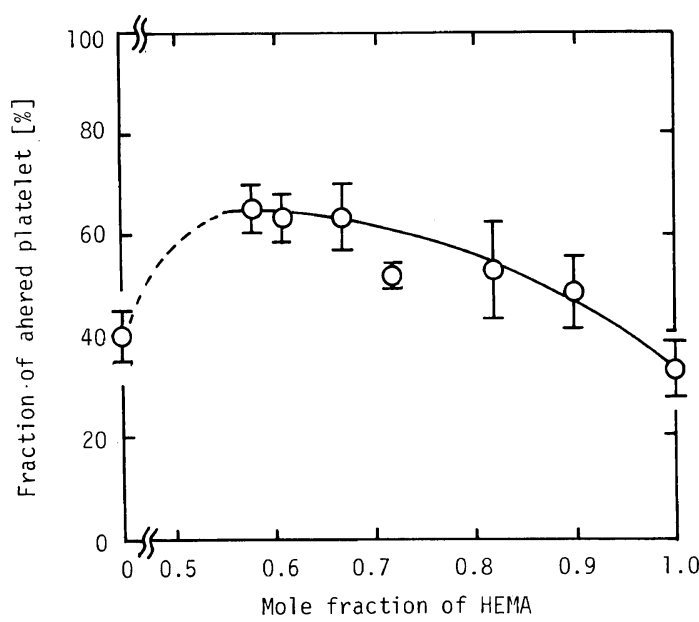

Figure 3. Platelet adhesion on the HEMA-DMS block copolymer surfaces. $[\%]=$ (number of platelets adhered on the polymer surface)/(number of platelets in whole blood) $\times 100$.

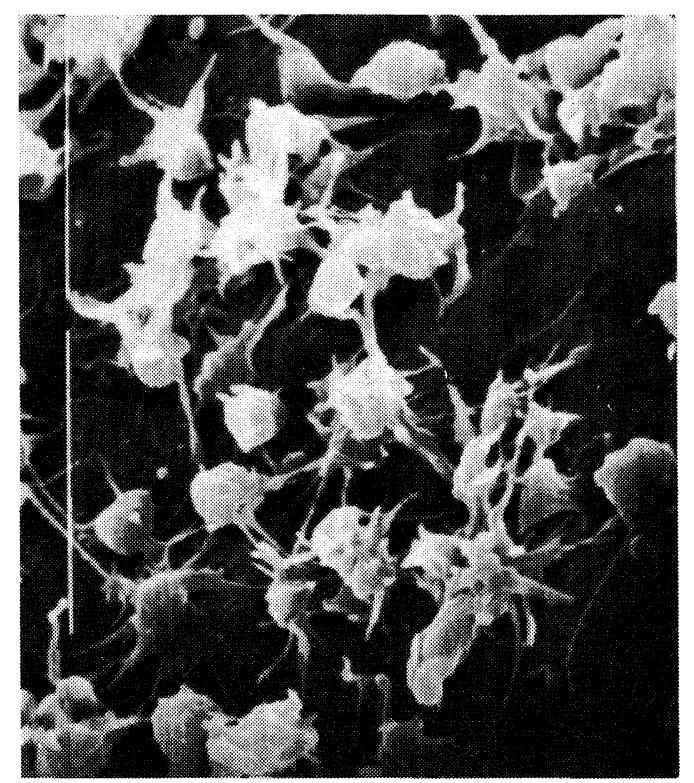

PHEMA
(Figure 4). The flattening of the adhered platelets on the PDMS surface implies that the interaction between the platelets and hydrophobic surface was strong. In contrast to the deformation of platelets observed on these homopolymers, the shape change of platelets and their aggregation were found to be suppressed effectively on the surface of block copolymers as shown in Figure 5. Particular, on the block copolymer containing the 0.67 mole fraction of HEMA [3], platelets were attached singly without aggregation or spreading.

Platelet adhesion increased on the microdomain surfaces of HEMA-DMS block copolymers as shown in Figure 3. It is not yet evident, however, whether this enhancement in platelet adhesion was due to the morphological change in the microdomains or merely a difference in the HEMA content in block copolymers. To clarify this problem, adhesion behaviour was investigated on the films of block copolymer cast from different solvents. The morphology of a microdomain could be altered by using different solvents even though an identical block copolymer was used. Figure 6 shows the results of platelet adhesion on the block co-

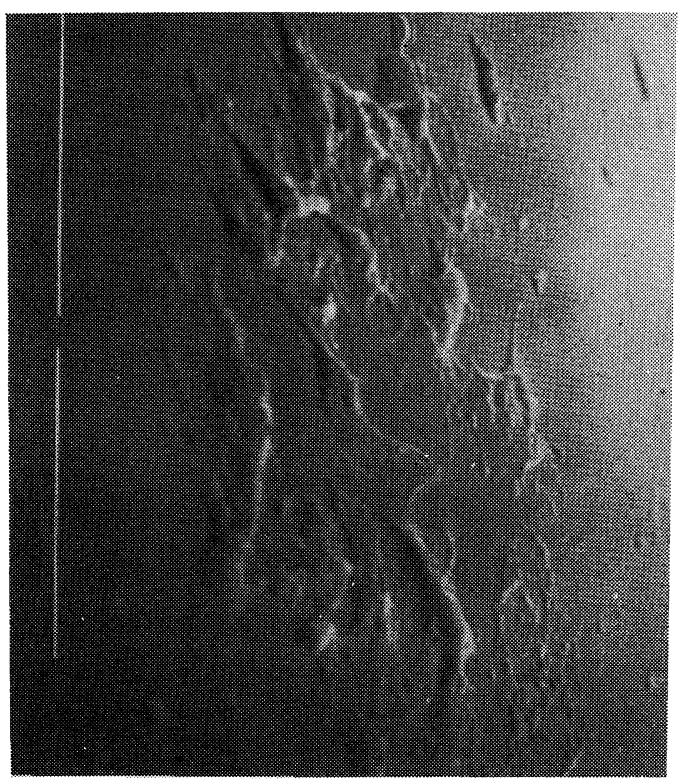

PDMS

Figure 4. Adhered platelets on the hydrophilic surface of PHEMA and hydrophobic surface of PDMS $(\times 4,700)$. 


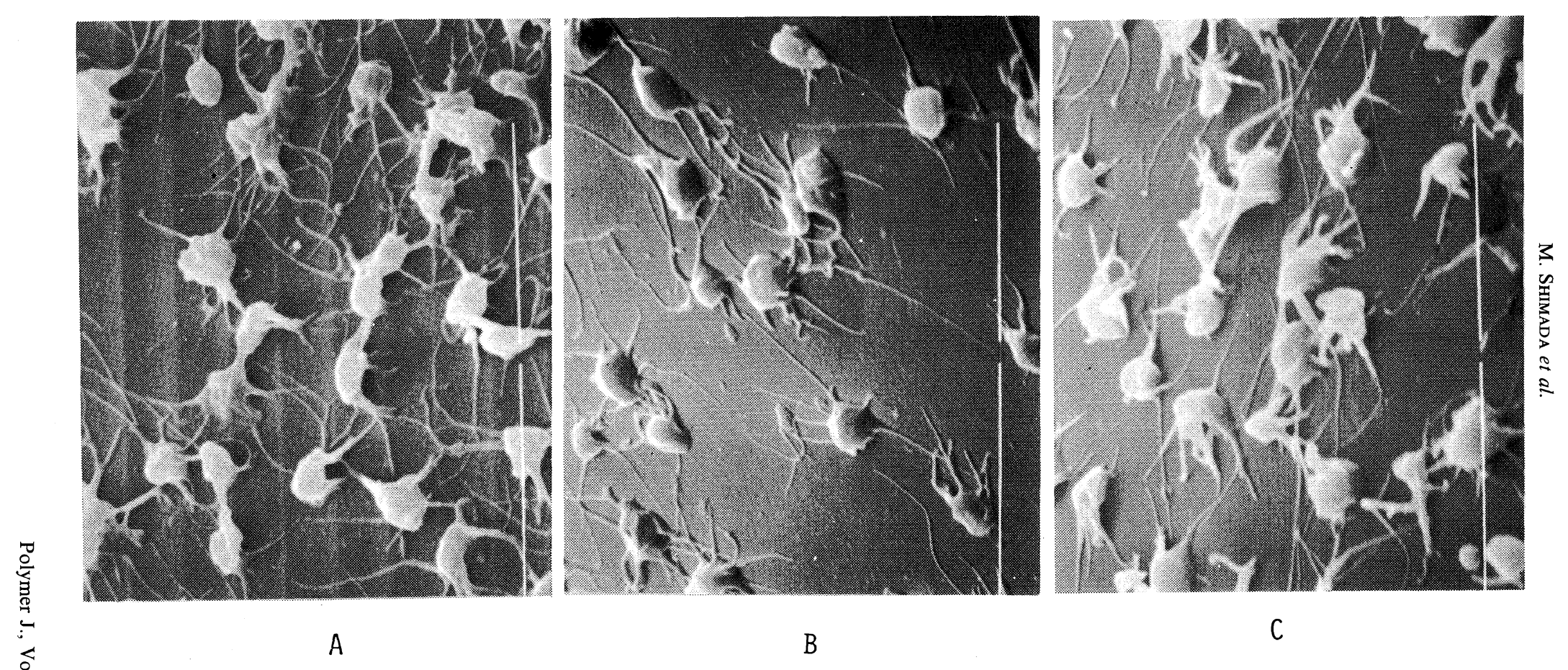

Figure 5. Adhered platelets on the HEMA-DMS block copolymer surfaces. Mole fraction of HEMA: A, (0.61) [2]; B, (0.67) [3]; C, $(0.82)[5](\times 4,700)$. 


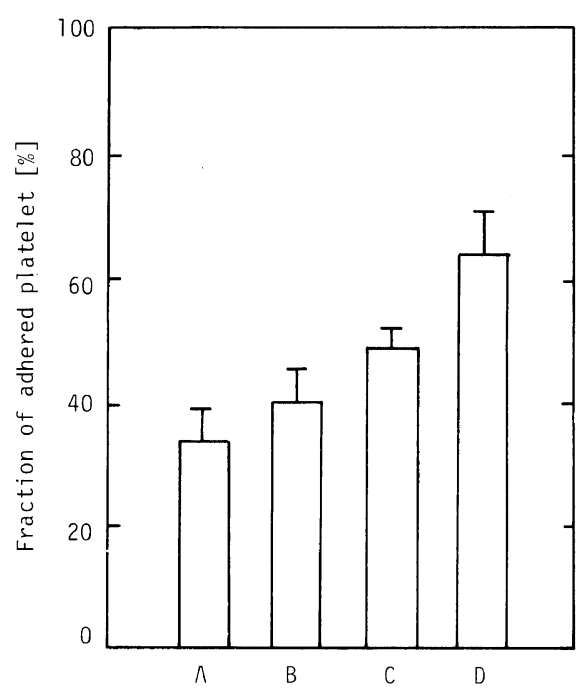

Figure 6. Effects of the morphology of the microphaseseparated structure of the HEMA-DMS block copolymer on platelet adhesion: A, PHEMA; B, PDMS; C, HEMA-DMS block copolymer [3] (0.67 mol fraction of HEMA) cast from pyridine; D, HEMA-DMS block copolymer [3] (0.67 mol fraction of HEMA) cast from DMF-toluene $(4: 6)$.

polymer surfaces of films cast from different solvents of DMF-toluene $(4: 6)$ and pyridine, compared with the case of PHEMA and PDMS surfaces. The surface of the film cast from a pyridine solutions revealed lower platelet adhesion than that cast from a DMF-toluene $(4: 6)$ solution. The microdomain structure of the block copolymer [3] exhibited different patterns of morphology with different casting solvents, as shown in Figures 1-B and 2. Despite the same composition of HEMA, the amount of adhered platelets changed corresponding to alteration in the morphology of the microdomains. Deformation of platelets was also suppressed on both surfaces of the films cast from DMF-toluene and pyridine solutions. From these results, the enhancement of platelet adhesion observed on the HEMA-DMS block copolymer was influenced predominantly by the morphology of the microdomains.

When a polymer surface is in contact with blood, adsorption of plasma proteins occurs immediately. ${ }^{10}$ Thus, the interaction of platelets with polymer is influenced by the nature of adsorbed layer of plasma proteins, e.g., amount, species, distribution, etc. We previously examined the adsorption phenomena of plasma proteins on the surface of HEMA-St block copolymer and found that the hydrophilic domains of HEMA adsorbed albumin, whereas the hydrophobic domains of styrene adsorbed $\gamma$-globulin or fibrinogen selectively. ${ }^{11}$ The adsorbed proteins thus form microphase-separated layers having an "organized structure" corresponding to the microdomain structure of the block copolymer surface. On the surface of HEMA-DMS block copolymers, it is also probable that this "organized structure" protein layer depending on protein species and amount is formed since the HEMA-DMS block copolymer exhibits a microphase-separated structure having hydrophilichydrophobic properties like that of HEMA-St block copolymer. ${ }^{2}$ We assume that the "organized structure" of the layer of adsorbed plasma proteins prevents shape change of the platelets by regulating the redistribution of glycoproteins situated in the plasma membranes of the platelets. ${ }^{12}$

We found that the HEMA-St block copolymers suppressed platelet adhesion, an opposite effect observed on the HEMA-DMS block copolymer surfaces. ${ }^{2}$ The surface free energy gap of the microdomains of the HEMA-DMS block copolymers is larger than that of the HEMA-St block copolymers, and hence the composition and amount of adsorbed proteins in the "organized structure" layers on the HEMA-DMS block copolymer surfaces may differ from those of the HEMA-St block copolymers. We consider that the composition and amount of adsorbed proteins influences platelet adhesion on the HEMA-DMS and HEMA-St block copolymer surfaces.

Acknowledgements. This research was supported by the Ministry of Education, Japan (Special Project Research, Design of Multiphase Biomedical Materials), the Asahi Glass Foundation for Industrial Technology and Japan Research Promotion Society for Cardiovascular Diseases.

\section{REFERENCES}

1. Y. Sakurai, T. Akaike, K. Kataoka, and T. Okano, "Biomedical Polymers," Academic Press, New York, 1980.

2. T. Okano, S. Nishiyama, I. Shinohara, T. Akaike, Y. Sakurai, K. Kataoka, and T. Tsuruta, J. Biomed. 
Mater. Res., 15, 393 (1981).

3. T. Okano, M. Katayama, and I. Shinohara, J. Appl. Polym. Sci., 22, 369 (1978).

4. T. Okano, K. Kataoka, Y. Sakurai, M. Shimada, M. Miyahara, T. Akaike, and I. Shinohara, Artif. Organs, 5, 468 (1981).

5. T. Okano, M. Katayama, S. Mogi, and I. Shinohara, Nippon Kagaku Kaishi, 1, 88 (1977).

6. J. M. Braun and J. E. Guillet, J. Polym. Sci., Polym. Chem. Ed., 14, 1073 (1976).

7. K. Kataoka, T. Akaike, Y. Sakurai, and T. Tsuruta, Makromol. Chem., 179, 1121 (1978).
8. G. Brecher and E. P. Cronkite, J. Appl. Phys., 3, 365 (1950).

9. T. Okano, M. Shimada, I. Shinohara, K. Kataoka, T. Akaike, and Y. Sakurai, "Biomaterials 1980," John Wiley and Sons, London, 1982, p 445.

10. R. Baier, Ann. New York Acad. Sci., 283, 17 (1977).

11. T. Okano, S. Nishiyama, I. Shinohara, T. Akaike, and Y. Sakurai, Polym. J., 10, 223 (1978).

12. K. Kataoka, T. Okano, T. Akaike, Y. Sakurai, M. Maeda, T. Nishimura, Y. Nitadori, T. Tsuruta, M. Shimada, and I. Shinohara, "Biomaterials 1980," John Wiley and Sons, London, 1982, p. 493. 